\title{
Exceptional geometry and Borcherds superalgebras
}

\section{Jakob Palmkvist}

Mitchell Institute for Fundamental Physics and Astronomy, Texas A\&M University, College Station, TX rr843, U.S.A.

E-mail: jakobpalmkvist@tamu.edu

ABSTRACT: We study generalized diffeomorphisms in exceptional geometry with U-duality group $E_{n(n)}$ from an algebraic point of view. By extending the Lie algebra $\mathfrak{e}_{n}$ to an infinitedimensional Borcherds superalgebra, involving also the extension to $\mathfrak{e}_{n+1}$, the generalized Lie derivatives can be expressed in a simple way, and the expressions take the same form for any $n \leqslant 7$. The closure of the transformations then follows from the Jacobi identity and the grading of $\mathfrak{e}_{n+1}$ with respect to $\mathfrak{e}_{n}$.

KeYwords: Supergravity Models, M-Theory, Differential and Algebraic Geometry, SpaceTime Symmetries

ARXIV EPRINT: 1507.08828 


\section{Contents}

1 Introduction 1

2 The algebras $\quad 2$

2.1 From $\mathfrak{e}_{n}$ to $\mathscr{B}_{n}$ and $\mathfrak{e}_{n+1}$, and further to $\mathscr{B}_{n+1} \quad 3$

2.2 Level decompositions 4

$\begin{array}{lll}2.3 & \text { Commutation relations } & 6\end{array}$

$\begin{array}{llr}3 & \text { The generalized diffeomorphisms } & 7\end{array}$

$\begin{array}{ll}3.1 \text { The section condition } & 7\end{array}$

3.2 Expressions for the generalized Lie derivative 8

$\begin{array}{lll}3.3 \text { Closure } & 10\end{array}$

$\begin{array}{lll}3.4 & \text { Reducibility } & 13\end{array}$

4 Discussion $\quad 14$

\section{Introduction}

Exceptional geometry generalizes ordinary geometry in eleven-dimensional supergravity, or M-theory, compactified to $D=(11-n)$ dimensions. In this generalization the $n$ dimensional internal tangent space, considered as a vector module of $\mathrm{GL}(n)$, is extended to an irreducible module of the U-duality group $E_{n(n)}$. All bosonic degrees of freedom are unified into a generalized metric, and the ordinary diffeomorphisms are unified with tensor gauge transformations into generalized diffeomorphisms [1-21].

The idea presented in this paper is to consider the $E_{n(n)}$ module as an odd subspace of a Borcherds superalgebra $\mathscr{B}_{n}$, which is an infinite-dimensional extension of the Lie algebra $\mathfrak{e}_{n}$, related to the ordinary Lie algebra extension $\mathfrak{e}_{n+1}$. By further extending $\mathscr{B}_{n}$ and $\mathfrak{e}_{n+1}$ to the Borcherds superalgebra $\mathscr{B}_{n+1}$ we find simple algebraic expressions for the generalized diffeomorphisms which take the same form for any $n \leqslant 7$. The closure of the transformations then follows from the Jacobi identity in $\mathscr{B}_{n+1}$ and the $\mathbb{Z}$-grading of $\mathfrak{e}_{n+1}$ with respect to $\mathfrak{e}_{n}$.

It is well known already that the level decomposition of the Borcherds superalgebra $\mathscr{B}_{n}$ with respect to the $\mathfrak{e}_{n}$ subalgebra gives the correct spectrum of $p$-forms in maximal $D$-dimensional supergravity, including all duals of lower rank fields, and also all additional $(D-1)$ - and $D$-forms potentials allowed by supersymmetry [22-27]. All their equations of motion and Bianchi identities can be expressed as one single Maurer-Cartan equation using the Borcherds superalgebra $\mathscr{B}_{n}[22]$, and this result can furthermore be generalized to gauged supergravity $[28,29]$, modifying $\mathscr{B}_{n}$ to a tensor hierarchy algebra [30]. The spectrum can also be derived from the indefinite Kac-Moody algebra $\mathfrak{e}_{11}$ [31-34] in accordance 
with the description of M-theory as a nonlinear realization of $\mathfrak{e}_{11}$ [35]. The correspondence between $\mathscr{B}_{n}$ and $\mathfrak{e}_{11}$ has been studied in [25, 36], and generalized to other Borcherds superalgebras and Kac-Moody algebras in [29, 37].

The appearance of the Borcherds superalgebras $\mathscr{B}_{n}$ in the context of exceptional geometry was observed in [38], where it was shown that the generalized diffeomorphisms are reducible, and lead to an infinite tower of ghosts for ghosts. The corresponding infinite sequence of $\mathfrak{e}_{n}$-representations agrees precisely with the level decomposition of $\mathscr{B}_{n}$ for positive levels, which was later explained in [39]. The same representations also appear in the tensor hierarchies considered in [16-18], related to those appearing in gauged supergravity [40, 41].

In this paper we show that the Borcherds structure is in fact hidden already in the generalized diffeomorphisms themselves, and not only in their reducibility. The correspondence with the ghost structure can then be shown directly, without using the results in [39]. Our results may lead to a way of including gravitational degrees of freedom in the Borcherds approach to supergravity, as well as to deeper insights into exceptional geometry. Since our results are generic for $n \leqslant 7$ they may provide some guidance in dealing with the difficulties associated to the dual graviton in the case $n=8[18,20,21]$, and in proceeding to $n \geqslant 9$. Unless otherwise stated, $3 \leqslant n \leqslant 7$ in this paper, but we will also comment on the case $n=8$.

The paper is organized as follows. In section 2 we describe the algebras that we will use, and how they are related to each other. In section 3 we first review the construction of the generalized diffeomorphisms given in [38] and then present our new algebraic expressions. To derive the identities needed for closure of the transformations we need to introduce the concept of generalized Jordan triple systems, and define a superversion of it. In the end of section 3 we tentatively discuss the reducibility of the transformations in view of our new results, and we continue the discussion in section 4 , where we also point out some natural directions for further research.

\section{The algebras}

In this section we will define the Borcherds superalgebra $\mathscr{B}_{n}$ as an extension of the Lie algebra $\mathfrak{e}_{n}$, and describe how both $\mathscr{B}_{n}$ and $\mathfrak{e}_{n+1}$ can be further extended to $\mathscr{B}_{n+1}$. Borcherds (super)algebras are generalizations of Kac-Moody (super)algebras and can themselves be further generalized to contragredient Lie (super)algebras. We will only consider the special cases that we are interested in here, and refer to [42-45] for details and general definitions.

We will assume the base field to be the real numbers, so that we get the split real forms of the corresponding complex algebras, since these are the ones that appear in the physical applications that we are interested in. From a purely mathematical point of view, we can equally well let the algebras remain complex.

We recall that $\mathfrak{e}_{n}$, as a special case of a Kac-Moody algebra, is defined as the Lie algebra generated by $3 n$ elements $e_{i}, f_{i}$ and $h_{i}=\left[e_{i}, f_{i}\right](i=1,2, \ldots, n)$ modulo the Chevalley-Serre relations

$$
\left[h_{i}, e_{j}\right]=a_{i j} e_{j}, \quad\left[h_{i}, f_{j}\right]=-a_{i j} f_{j}, \quad\left[e_{i}, f_{j}\right]=\delta_{i j} h_{j},
$$




$$
\left(\operatorname{ad} e_{i}\right)^{1-a_{i j}}\left(e_{j}\right)=\left(\operatorname{ad} f_{i}\right)^{1-a_{i j}}\left(f_{j}\right)=0 \quad(i \neq j),
$$

where the Cartan matrix $a_{i j}$ is given by the following Dynkin diagram.

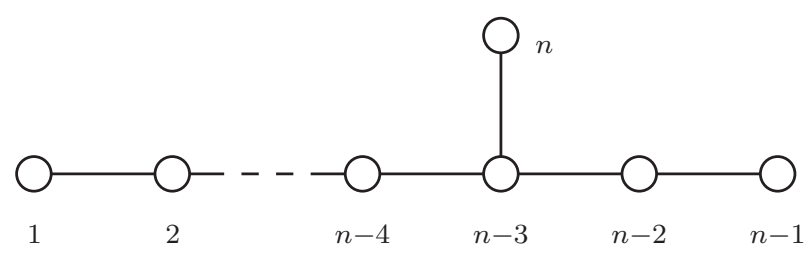

The nodes represent simple roots $\alpha_{i}$ which we normalize by $\left(\alpha_{i}, \alpha_{i}\right)=2$. Their mutual inner products are then either $\left(\alpha_{i}, \alpha_{j}\right)=-1$ or $\left(\alpha_{i}, \alpha_{j}\right)=0$, in such a way that the nodes $i$ and $j$ are connected by $\left|\left(\alpha_{i}, \alpha_{j}\right)\right|$ lines, and the Cartan matrix is then given by

$$
a_{i j}=\left(\alpha_{i}, \alpha_{j}\right)
$$

In this paper we only consider the cases $3 \leqslant n \leqslant 8$ (and if not $n=8$ is stated explicitly we assume $3 \leqslant n \leqslant 7$ ). For $n=5$ and $n=4$, the Lie algebras $\mathfrak{e}_{n}$ are not exceptional, but coincide with the classical Lie algebras $\mathfrak{d}_{5}=\mathfrak{s o}(5,5)$ and $\mathfrak{a}_{4}=\mathfrak{s l}(5)$, respectively. For $n=3$ the Lie algebra is not even simple but equal to the direct sum of $\mathfrak{a}_{1}=\mathfrak{s l}(2)$ and $\mathfrak{a}_{2}=\mathfrak{s l}(3)$.

\subsection{From $\mathfrak{e}_{n}$ to $\mathscr{B}_{n}$ and $\mathfrak{e}_{n+1}$, and further to $\mathscr{B}_{n+1}$}

By adding a node to the Dynkin diagram, $\mathfrak{e}_{n}$ can be extended to either $\mathfrak{e}_{n+1}$ or to the Borcherds superalgebra $\mathscr{B}_{n}$, depending on whether the node is white (like the orginal nodes in the $\mathfrak{e}_{n}$ diagram) or gray $(\otimes)$ as illustrated below, and as we will now explain. The additional node represents an additional simple root which we denote by $\alpha_{0}$ in the $\mathfrak{e}_{n+1}$ case, and by $\beta_{0}$ in the $\mathscr{B}_{n}$ case. They have the same inner product with the simple root $\alpha_{1}$ of the $\mathfrak{e}_{n}$ subalgebra,

$$
\left(\alpha_{0}, \alpha_{1}\right)=\left(\beta_{0}, \alpha_{1}\right)=-1
$$

and are orthogonal to all other simple roots of $\mathfrak{e}_{n}$. However, like the original simple roots of the $\mathfrak{e}_{n}$ subalgebra, $\alpha_{0}$ has norm squared equal to two, $\left(\alpha_{0}, \alpha_{0}\right)=2$, whereas $\beta_{0}$ is a null root, $\left(\beta_{0}, \beta_{0}\right)=0$. Furthermore, $\alpha_{0}$ is even whereas $\beta_{0}$ is odd, which means that the Chevalley generators $e_{0}$ and $f_{0}$ associated to $\beta_{0}$ are odd elements in the Lie superalgebra $\mathscr{B}_{n}$ (so that, for example, $\left[e_{0}, f_{0}\right]=\left[f_{0}, e_{0}\right]$ instead of $\left.\left[e_{0}, f_{0}\right]=-\left[f_{0}, e_{0}\right]\right)$. Both $\mathfrak{e}_{n+1}$ and $\mathscr{B}_{n}$ are then defined by the same Chevalley-Serre relations as before, (2.1)-(2.2), with the Cartan matrix still given by (2.3), but now including also the additional simple root $\alpha_{0}$ or $\beta_{0}$. 


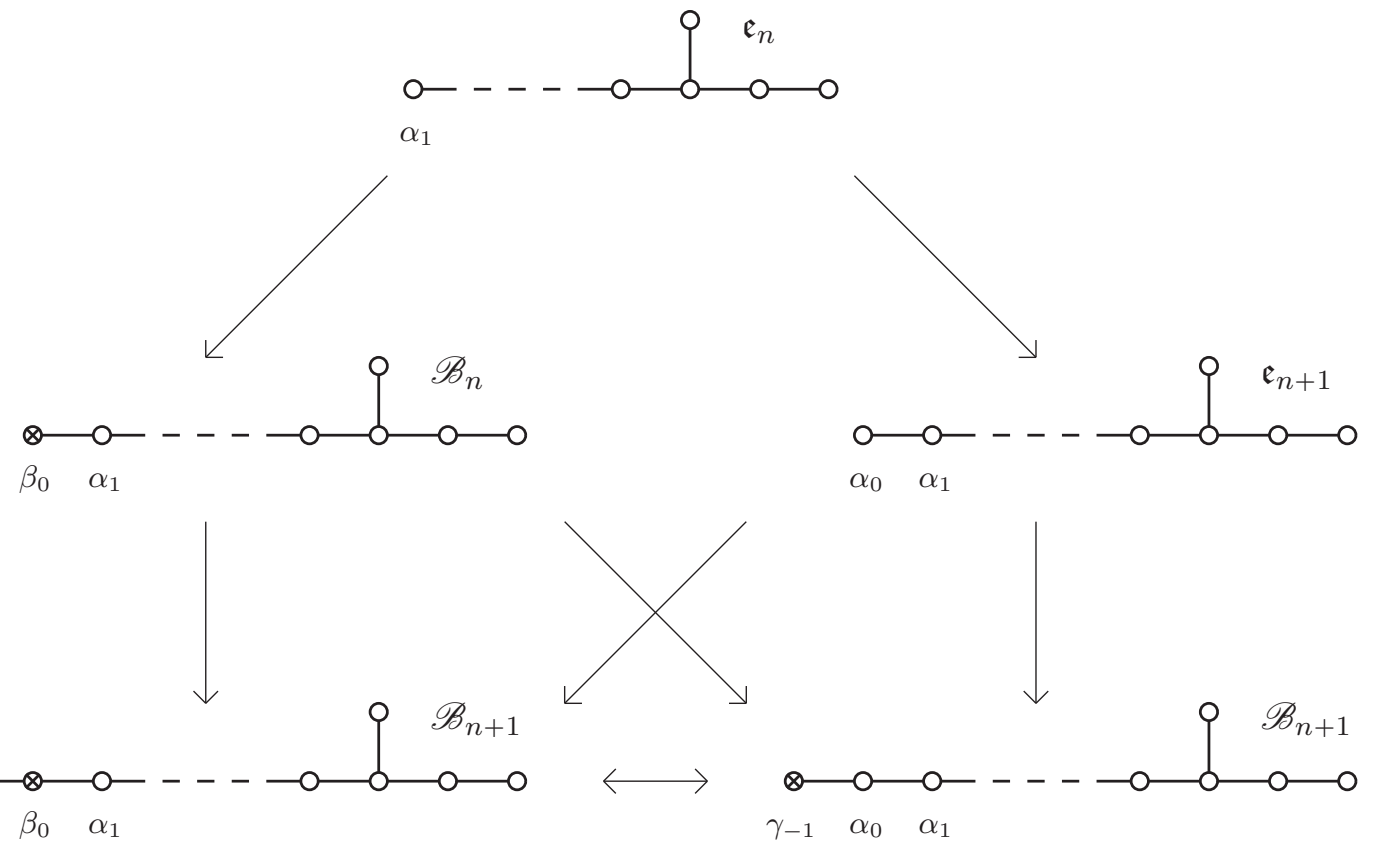

Both $\mathfrak{e}_{n+1}$ and $\mathscr{B}_{n}$ can in turn be extended to the Borcherds superalgebra $\mathscr{B}_{n+1}$, as illustrated above. When we extend $\mathscr{B}_{n}$ to $\mathscr{B}_{n+1}$ (left vertical arrow) we add another odd null root $\beta_{-1}$ to the set of simple roots, such that $\left(\beta_{-1}, \beta_{0}\right)=1$ (note the sign!) and $\beta_{-1}$ is orthogonal to all the simple roots of $\mathfrak{e}_{n}$. The embedding of $\mathfrak{e}_{n+1}$ in $\mathscr{B}_{n+1}$ is then given by identifying $\alpha_{0}$ in $\mathfrak{e}_{n+1}$ with $\left(\beta_{-1}+\beta_{0}\right)$ in $\mathscr{B}_{n+1}$ (diagonal arrow going to the left). Alternatively, we could extend $\mathfrak{e}_{n+1}$ to $\mathscr{B}_{n+1}$ by adding an odd null root $\gamma_{-1}$ such that $\left(\gamma_{-1}, \alpha_{0}\right)=-1$ and $\gamma_{-1}$ is orthogonal to the simple roots of $\mathfrak{e}_{n}$. The embedding of $\mathscr{B}_{n}$ in $\mathscr{B}_{n+1}$ would then be given by identifying $\beta_{0}$ in $\mathscr{B}_{n}$ with $\left(\gamma_{-1}+\alpha_{0}\right)$ in $\mathscr{B}_{n+1}$, as described in [46]. The two different Dynkin diagrams of $\mathscr{B}_{n+1}$ are related by a so-called odd reflection mapping $\gamma_{-1}$ and $-\beta_{-1}$ to each other $[29,46]$. We choose the former approach here, corresponding to the left Dynkin diagram of $\mathscr{B}_{n+1}$, so that the subscripts 0 and -1 of any Chevalley generators always refer to $\beta_{0}$ and $\beta_{-1}$.

The Killing form on $\mathfrak{e}_{n}$ can be extended to a supersymmetric invariant bilinear form on the whole of $\mathscr{B}_{n+1}$, which we denote by $\langle x \mid y\rangle$ for any two elements $x$ and $y$. Supersymmetry here means $\langle x \mid y\rangle=-\langle y \mid x\rangle$ if both elements are odd, and $\langle x \mid y\rangle=\langle y \mid x\rangle$ if at least one of them is even. Invariance always means $\langle[x, y] \mid z\rangle=\langle x \mid[y, z]\rangle$.

\subsection{Level decompositions}

The extension of $\mathfrak{e}_{n}$ by the additional simple root $\beta_{0}$ gives rise to a $\mathbb{Z}$-grading, or level decomposition, of $\mathscr{B}_{n}$ with $e_{0}$ and $f_{0}$ at level +1 and -1 , respectively, and the $\mathfrak{e}_{n}$ subalgebra at level zero (together with the Cartan element $h_{0}=\left[e_{0}, f_{0}\right]$ ). For any integer $p$ we denote the subspace of $\mathscr{B}_{n}$ at level $p$ by $\mathscr{U}_{p}$, so that $\left[\mathscr{U}_{p}, \mathscr{U}_{q}\right]=\mathscr{U}_{p+q}$, and $\mathscr{B}_{n}$ is the direct sum of all these subspaces. In the same way, the extension of $\mathfrak{e}_{n}$ by the additional simple root $\alpha_{0}$ gives rise to a level decomposition of $\mathfrak{e}_{n+1}$ for which we denote the subspace at level $p$ by $\tilde{\mathscr{U}}_{p}$.

The vector spaces $\mathscr{U}_{p}$ and $\tilde{\mathscr{U}}_{p}$ are also modules for $\mathfrak{e}_{n}$-representations $R_{p}$ and $\tilde{R}_{p}$, respectively, given by the adjoint action of the $\mathfrak{e}_{n}$ subalgebra at level zero. While $R_{1}=\tilde{R}_{1}$, 
the representations $R_{2}$ and $\tilde{R}_{2}$ are different, contained in the symmetric and antisymmetric parts, respectively, of the tensor product $R_{1} \times R_{1}$ (since $\mathscr{U}_{1}$ is an odd subspace of the Lie superalgebra $\mathscr{B}_{n}$, and $\mathfrak{e}_{n+1}$ is an ordinary Lie algebra). For $n \leqslant 6$ the Lie algebra $\mathfrak{e}_{n+1}$ is 3-graded with respect to $\mathfrak{e}_{n}$, which means that $\tilde{\mathscr{U}}_{p}=0$ for $|p| \geqslant 2$, and thus $\tilde{R}_{2}$ vanishes. In the case $n=7$ we instead have a 5 -grading, $\tilde{\mathscr{U}}_{p}=0$ for $|p| \geqslant 3$, where the subspaces $\tilde{\mathscr{U}}_{ \pm 2}$ are one-dimensional, so that $\tilde{R}_{2}=\mathbf{1}$. For $n=8$, the extended Lie algebra $\mathfrak{e}_{n+1}=\mathfrak{e}_{9}$ is the affine extension of $\mathfrak{e}_{8}$ with infinitely many subspaces $\tilde{\mathscr{U}}_{p}$, and $\tilde{R}_{p}$ is equal to the adjoint representation 248 of $\mathfrak{e}_{8}$ for any $p \neq 0$. (Usually in the definition of $\mathfrak{e}_{9}$, or any other affine Kac-Moody algebra, a basis element called derivation is included at level zero, in addition to those in the definition of $\mathfrak{e}_{n+1}$ above. It can be identified with $h_{-1}$ in the further extension to $\mathscr{B}_{n+1}=\mathscr{B}_{9}$.) On the other hand, $\mathscr{B}_{n}$ is infinite-dimensional for all $n$, and the dimensions of the infinitely many (possibly reducible) representations $R_{p}$ grow with the level $p \geqslant 1$. For any $p$, the representations $R_{p}$ and $R_{-p}$ (or $\tilde{R}_{p}$ and $\tilde{R}_{-p}$ ) are conjugate to each other.

Clearly $R_{1}$ is an irreducible representation with a lowest weight vector $e_{0}$ and lowest weight $-\Lambda^{1}$, where the fundamental weights $\Lambda^{i}$ are defined by $\left(\alpha_{i}, \Lambda^{j}\right)=\delta_{i}{ }^{j}$. The dimension of $R_{1}$ is $6,10,16,27,56,248$ for $n=3,4,5,6,7,8$, respectively. The representation $R_{2}$ is irreducible for $n \leqslant 7$ with lowest weight $-\Lambda^{n-1}$. For $n=8$, it decomposes into a direct sum of a representation with lowest weight $-\Lambda^{n-1}$ and an additional singlet. Tables with the representations $R_{p}$ for all $n$ and the first few positive levels $p$ can be found in for example [29], and an efficient recursive method to compute them for all positive levels was given in [39]. In section 3.4 we will see that the sequence of representations $R_{p}$ for $p \geqslant 1$ is related to the infinite reducibility of the generalized diffeomorphisms in exceptional geometry, as was observed in [38].

Each subspace $\mathscr{U}_{p}$ in the level decomposition of $\mathscr{B}_{n}$ with respect to the gray node can in turn be further decomposed with respect to any of the other nodes. Choosing node $n$ (referring to the Dynkin diagram in the beginning of this section), we then write $\mathscr{U}_{ \pm 1}=\mathscr{U}_{ \pm 1}^{0} \oplus \mathscr{U}_{ \pm 1}{ }^{ \pm}$where $\mathscr{U}_{ \pm 1}^{0}$ is an $n$-dimensional subspace spanned by root vectors for which the roots have zero coefficients corresponding to $\alpha_{n}$ in the basis of simple roots, and $\mathscr{U}_{ \pm 1} \pm$ is spanned by root vectors for which the sign of this coefficient is \pm 1 . The subalgebra of $\mathscr{B}_{n}$ generated by $\mathscr{U}_{ \pm 1}{ }^{0}$ is the 3 -graded Lie superalgebra

$$
A(n-1,0)=\mathfrak{s l}(n \mid 1)=\mathscr{U}_{-1}{ }^{0} \oplus \mathfrak{g l}(n) \oplus \mathscr{U}_{1}^{0},
$$

obtained by removing node $n$ from the Dynkin diagram of $\mathscr{B}_{n}$. In the same way we write $\tilde{\mathscr{U}}_{ \pm 1}=\tilde{\mathscr{U}}_{ \pm 1}{ }^{0} \oplus \tilde{\mathscr{U}}_{ \pm 1}{ }^{ \pm}$, where the subalgebra of $\mathfrak{e}_{n+1}$ generated by $\tilde{\mathscr{U}}_{ \pm 1}{ }^{0}$ is the 3 -graded Lie algebra

$$
\mathfrak{a}_{n}=\mathfrak{s l}(n+1)=\tilde{\mathscr{U}}_{-1}^{0} \oplus \mathfrak{g l}(n) \oplus \tilde{\mathscr{U}}_{1}^{0} .
$$

The adjoint action of the subalgebra $\mathfrak{g l}(n)$ at level zero on $\mathscr{U}_{1}^{0}$ and $\tilde{\mathscr{U}}_{1}^{0}$ is given by the $n$-dimensional vector (or fundamental) representation.

Thus the restriction of $\mathscr{U}_{1}$ to $\mathscr{U}_{1}^{0}$ at level one in $\mathscr{B}_{n}$ leads to the restriction of $\mathfrak{e}_{n} \oplus \mathbb{R}$ to $\mathfrak{g l}(n)$ at level zero, which means reducing exceptional geometry to ordinary geometry (as will be more clear in the next section). For this reason, we use indices

$$
m, n, \ldots=1,2, \ldots, n, \quad \mathcal{M}, \mathfrak{N}, \ldots=1,2, \ldots, \operatorname{dim} R_{1},
$$


and let $E_{\mathscr{M}}$ be a basis of $\mathscr{U}_{1}$ such that $E_{m}$ is a basis of $\mathscr{U}_{1}^{0}$. We then let $F^{\mathscr{M}}$ be a basis of $\mathscr{U}_{-1}$ such that

$$
\left\langle E_{\mathscr{M}} \mid F^{\mathcal{N}}\right\rangle=-\left\langle F^{\mathcal{N}} \mid E_{\mathscr{M}}\right\rangle=\delta_{\mathscr{M}^{\mathcal{X}}},
$$

which implies that $F^{m}$ is a basis of $\mathscr{U}_{-1}{ }^{0}$. Considering $\mathfrak{e}_{n+1}$ as a subalgebra of $\mathscr{B}_{n+1}$ these bases of $\mathscr{U}_{ \pm 1}$ give rise to corresponding bases $\tilde{E}_{\mathscr{M}}=\left[e_{-1}, E_{\mathscr{M}}\right]$ and $\tilde{F}^{\mathscr{M}}=-\left[f_{-1}, F^{\mathscr{M}}\right]$ of $\tilde{\mathscr{U}}_{ \pm 1}$ such that

$$
\left\langle\tilde{E}_{\mathscr{M}} \mid \tilde{F}^{\mathcal{X}}\right\rangle=\left\langle\tilde{F}^{\mathcal{X}} \mid \tilde{E}_{\mathscr{M}}\right\rangle=\delta_{\mathscr{M}}{ }^{\mathcal{N}} .
$$

\subsection{Commutation relations}

The commutation relations in $\mathscr{B}_{n}$ and $\mathfrak{e}_{n+1}$ of the elements at level \pm 1 with each other and with those at level zero were given in [36]. We let $t_{\alpha}$ be a basis of $\mathfrak{e}_{n}$, raise the adjoint $\mathfrak{e}_{n}$ index ${ }_{\alpha}$ with the inverse of the Killing form, (so that $\left\langle t_{\alpha} \mid t^{\beta}\right\rangle=\delta_{\alpha}{ }^{\beta}$ ), and introduce the Cartan elements

$$
h=(9-n) h_{0}+(10-n) h_{1}+\cdots+6 h_{n-3}+4 h_{n-2}+2 h_{n-1}+3 h_{n}
$$

and $\tilde{h}=h+(9-n) h_{-1}$, which span the orthogonal complements to $\mathfrak{e}_{n}$ at level zero in $\mathscr{B}_{n}$ and $\mathfrak{e}_{n+1}$, respectively (so that $\left[t_{\alpha}, h\right]=\left[t_{\alpha}, \tilde{h}\right]=0$ ). Then the relations are

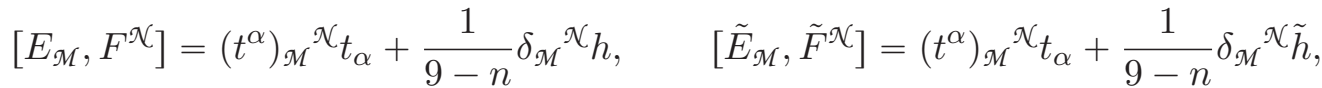

$$
\begin{aligned}
& {\left[t_{\alpha}, E_{\mathscr{M}}\right]=\left(t_{\alpha}\right)_{\mathscr{M}^{\mathcal{N}}} E_{\mathcal{N}}, \quad\left[t_{\alpha}, \tilde{E}_{\mathscr{M}}\right]=\left(t_{\alpha}\right)_{\mathcal{M}^{\mathcal{N}}} \tilde{E}_{\mathscr{N}},} \\
& {\left[t_{\alpha}, F^{\mathcal{X}}\right]=-\left(t_{\alpha}\right)_{\mathcal{M}} \mathcal{X}^{\mathcal{M}}, \quad\left[t_{\alpha}, \tilde{F}^{\mathcal{X}}\right]=-\left(t_{\alpha}\right)_{\mathcal{M}^{\mathcal{X}}} \tilde{F}^{\mathscr{M}},} \\
& {\left[h, E_{\mathscr{M}}\right]=-(10-n) E_{\mathscr{M}}, \quad\left[\quad\left[\tilde{h}_{\mathscr{E}_{\mathcal{M}}}\right]=(8-n) \tilde{E}_{\mathscr{M}},\right.} \\
& {\left[h, F^{\mathcal{X}}\right]=(10-n) F^{\mathcal{X}}, \quad\left[\tilde{h}, \tilde{F}^{\mathcal{X}}\right]=-(8-n) \tilde{F}^{\mathcal{X}} \text {. }}
\end{aligned}
$$

Following [36] (but replacing $g$ there with $\tilde{f}$ ) we introduce the $\mathfrak{e}_{n}$ invariant tensors

$$
f_{\mathcal{M}^{\mathcal{X}}}{ }_{\mathcal{P}}^{Q}=\left\langle\left[\left[E_{\mathcal{M}}, F^{\mathcal{X}}\right], E_{\mathcal{P}}\right] \mid F^{Q}\right\rangle, \quad \tilde{f}_{\mathcal{M}^{\mathcal{N}}}{ }_{\mathcal{P}}^{Q}=\left\langle\left[\left[\tilde{E}_{\mathcal{M}}, \tilde{F}^{\mathcal{X}}\right], \tilde{E}_{\mathcal{P}}\right] \mid \tilde{F}^{Q}\right\rangle
$$

(which are in fact structure constants of generalized Jordan triple products, as we will see in section 3.3) and their (anti-)symmetrized versions

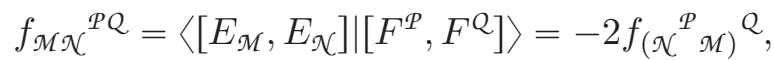

$$
\begin{aligned}
& \tilde{f}_{\mathscr{M} \mathcal{K}}{ }^{P Q}=\left\langle\left[\tilde{E}_{\mathscr{M}}, \tilde{E}_{\mathcal{N}}\right] \mid\left[\tilde{F}^{\mathcal{P}}, \tilde{F}^{Q}\right]\right\rangle=-2 \tilde{f}_{\left[\mathcal{N}^{P} \mathscr{M}\right]}{ }^{Q} .
\end{aligned}
$$

We thus have

$$
\begin{aligned}
& {\left[\left[E_{\mathcal{M}}, F^{\mathcal{N}}\right], E_{\mathcal{P}}\right]=f_{\mathcal{M}^{\mathcal{X}}}{ }^{Q} E_{Q}, \quad\left[\left[\tilde{E}_{\mathcal{M}}, \tilde{F}^{\mathcal{N}}\right], \tilde{E}_{\mathcal{P}}\right]=\tilde{f}_{\mathcal{M}} \mathcal{X}_{\mathcal{P}}{ }^{Q} \tilde{E}_{Q},} \\
& {\left[\left[E_{\mathscr{M}}, E_{\mathcal{N}}\right], F^{\mathcal{P}}\right]=f_{\mathscr{M} \mathcal{N}}{ }^{P Q} E_{Q}, \quad\left[\left[\tilde{E}_{\mathscr{M}}, \tilde{E}_{\mathcal{N}}\right], \tilde{F}^{\mathcal{P}}\right]=\tilde{f}_{\mathcal{M} \mathcal{K}}{ }^{P Q} \tilde{E}_{Q} \text {, }}
\end{aligned}
$$

and from (2.11) we get explicitly

$$
f_{\mathcal{M}^{\mathcal{N}}}{ }^{Q}{ }^{Q}=\left(t_{\alpha}\right)_{\mathcal{M}^{\mathcal{N}}}\left(t^{\alpha}\right)_{\mathcal{P}}{ }^{Q}-\frac{10-n}{9-n} \delta_{\mathcal{M}^{\mathcal{N}}} \delta_{\mathcal{P}}{ }^{Q}
$$




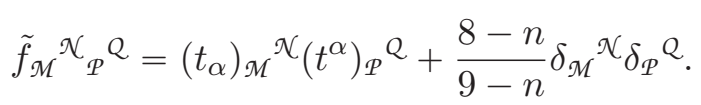

Restricting the basis elements to $\mathscr{U}_{ \pm 1}^{0}$ and $\tilde{\mathscr{U}}_{ \pm 1}{ }^{0}$ we get

$$
f_{m}{ }^{n} p^{q}=-\delta_{m}{ }^{n} \delta_{p}^{q}+\delta_{p}{ }^{n} \delta_{m}^{q} \quad \tilde{f}_{m}{ }^{n} p^{q}=\delta_{m}{ }^{n} \delta_{p}^{q}+\delta_{p}{ }^{n} \delta_{m}^{q} .
$$

In accordance with the 3 -gradings (2.5) and (2.6) we have

$$
\left[E_{m}, E_{n}\right]=\left[\tilde{E}_{m}, \tilde{E}_{n}\right]=\left[F^{m}, F^{n}\right]=\left[\tilde{F}^{m}, \tilde{F}^{n}\right]=0
$$

and thus

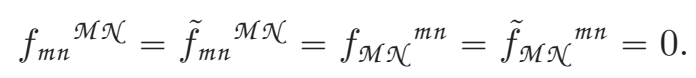

Considering $\mathscr{B}_{n}$ and $\mathfrak{e}_{n+1}$ as subalgebras of $\mathscr{B}_{n+1}$ we can generalize (2.14) to the set of relations

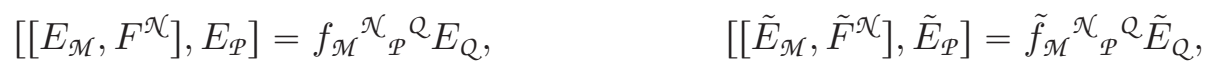

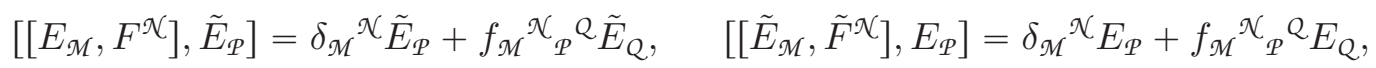

$$
\begin{aligned}
& {\left[\left[E_{\mathscr{M}}, \tilde{F}^{\mathcal{X}}\right], E_{\mathscr{P}}\right]=0, \quad\left[\left[\tilde{E}_{\mathcal{M}}, F^{\mathcal{N}}\right], \tilde{E}_{\mathcal{P}}\right]=0,} \\
& {\left[\left[E_{\mathscr{M}}, \tilde{F}^{\mathcal{N}}\right], \tilde{E}_{\mathscr{P}}\right]=\delta_{\mathscr{M}}{ }^{\mathcal{N}} E_{\mathcal{P}}, \quad\left[\left[\tilde{E}_{\mathscr{M}}, F^{\mathcal{N}}\right], E_{\mathcal{P}}\right]=-\delta_{\mathscr{M}}{ }^{\mathcal{N}} \tilde{E}_{\mathcal{P}},}
\end{aligned}
$$

which will be useful in the next section.

\section{The generalized diffeomorphisms}

We will now relate the algebraic concepts introduced in the preceding section to the context of eleven-dimensional supergravity (or M-theory) compactified to $D=(11-n)$ dimensions. At each point in the eleven-dimensional spacetime manifold, the $n$-dimensional subspace of the tangent space corresponding to the $n$ compactified dimensions can be considered as a vector module of $\mathfrak{g l}(n)$, which in turn can be extended to a module for the representation $R_{1}$ of $\mathfrak{e}_{n}$. The idea is to identify this $\mathfrak{e}_{n}$-module with the subspace $\mathscr{U}_{1}$, the $n$-dimensional subspace of $\mathscr{U}_{1}$ corresponding to the $n$ compactified dimensions with $\mathscr{U}_{1}^{0}$, and their coordinate bases with $E_{\mathcal{M}}$ and $E_{m}$, respectively. We furthermore ignore the remaining $D$ dimensions, and thus consider any vector field $V$ as an element in $\mathscr{U}_{1}$, expanded in the coordinate basis as $V=V^{\mathfrak{M}} E_{\mathscr{M}}$.

\subsection{The section condition}

We are interested in fields that only depend on the $n$ physical coordinates corresponding to the $\mathscr{U}_{1}^{0}$ subspace of $\mathscr{U}_{1}$. Any such field $A$ thus satisfies

$$
E_{\mathscr{M}} \in \mathscr{U}_{1}^{+} \quad \Rightarrow \quad \partial_{\mathscr{M}} A=0 .
$$

Because of (2.19) this implies the section condition

$$
f_{\mathcal{M} \mathcal{K}^{P Q}} \partial_{\mathcal{P}} \partial_{Q} A=f_{\mathcal{M} \mathcal{X}^{P Q}} \partial_{\mathcal{P}} A \partial_{Q} B=\tilde{f}_{\mathcal{M} \mathcal{X}^{P Q}} \partial_{\mathcal{P}} A \partial_{Q} B=0
$$


for any field $A$, or any pair of fields $A$ and $B$. Following [38] we write this as

$$
f_{\mathcal{M} \mathcal{K}^{P Q}}\left(\partial_{\mathcal{P}} \otimes \partial_{Q}\right)=\tilde{f}_{\mathcal{M} \mathcal{K}^{P Q}}\left(\partial_{\mathcal{P}} \otimes \partial_{Q}\right)=0,
$$

where $\left(\partial_{\mathcal{P}} \otimes \partial_{\mathcal{Q}}\right)$ denotes either $\partial_{\mathcal{P}} \partial_{\mathcal{Q}} A$ or $\partial_{\mathcal{P}} A \partial_{\mathcal{Q}} B$. We can also equivalently replace $f_{\mathcal{M} \mathcal{X}^{\mathcal{P Q}}}$

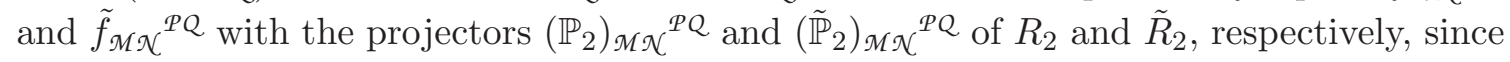
this only amounts to a rescaling of each irreducible part of these representations. The section condition then becomes

$$
\left(\mathbb{P}_{2}\right)_{\mathcal{M} \mathcal{X}^{\mathcal{Q}}}\left(\partial_{\mathcal{P}} \otimes \partial_{Q}\right)=\left(\tilde{\mathbb{P}}_{2}\right)_{\mathcal{M} \mathcal{K}}{ }^{P Q}\left(\partial_{\mathcal{P}} \otimes \partial_{Q}\right)=0
$$

Unlike the original constraint (3.1) the section condition (3.2) is $\mathfrak{e}_{n}$-covariant, and is implied not only by (3.1) but also by solutions equivalent to (3.1), where $\mathscr{U}_{1}^{+}$is replaced with different subspaces of $\mathscr{U}_{1}$ that can be mapped to $\mathscr{U}_{1}{ }^{+}$by $\mathfrak{e}_{n}$-transformations.

In addition there are solutions where the fields only depend on $(n-1)$ coordinates, corresponding to the subspace of $\mathscr{U}_{1}$ at level zero in a further decomposition with respect to node $(n-2)$, spanned by root vectors for which the roots have zero coefficients corresponding to $\alpha_{n-2}$ in the basis of simple roots (or equivalent solutions obtained by $\mathfrak{e}_{n^{-}}$ transformations). These solutions correspond to compactification of type IIB supergravity from ten to $(11-n)$ dimensions [16-18].

\subsection{Expressions for the generalized Lie derivative}

Under a generalized diffeomorphism generated by a vector field $U$, the transformation of another vector field $V$ is given by the generalized Lie derivative

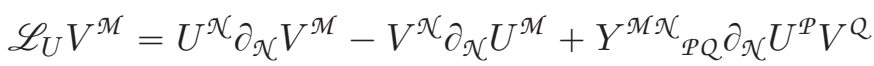

$$
\begin{aligned}
& =U^{\mathcal{N}} \partial_{\mathfrak{X}} V^{\mathscr{M}}+Z^{\mathfrak{M} \mathcal{X}}{ }_{\mathcal{Q Q}} \partial_{\mathcal{N}} U^{\mathcal{P}} V^{Q},
\end{aligned}
$$

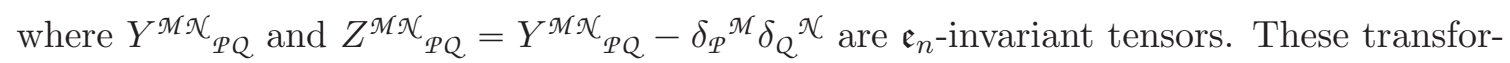
mations were defined in [8] and explicitly reconstructed from the $\mathfrak{e}_{n}$-covariant ansatz above in [38]. It was found in [38] that they close into an algebra according to

$$
\left[\mathscr{L}_{U}, \mathscr{L}_{V}\right]=\mathscr{L}_{\llbracket U, V \rrbracket}
$$

where $\llbracket U, V \rrbracket$ denotes the antisymmetrized generalized Lie derivative,

$$
\mathscr{L}_{\llbracket U, V \rrbracket}=\frac{1}{2}\left(\mathscr{L}_{U} V-\mathscr{L}_{V} U\right),
$$

if the tensor $Y$ satisfies the identities

$$
\begin{aligned}
& Y^{\mathcal{M} \mathcal{K}_{P Q}} \partial_{\mathcal{M}} \otimes \partial_{\mathcal{K}}=0,
\end{aligned}
$$

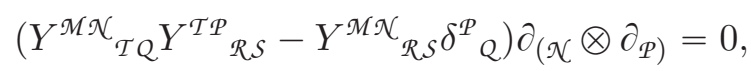

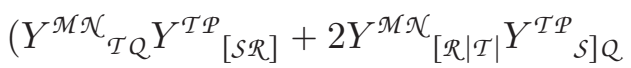

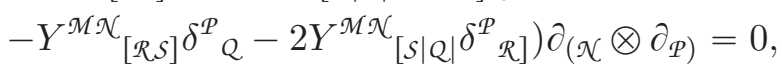




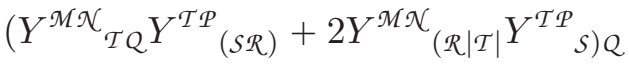

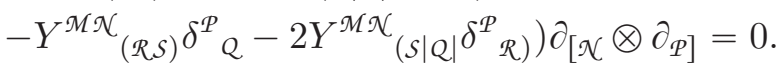

Up to the section condition (3.4), these identities uniquely determine the tensor $Y$, which in [38] was found to be

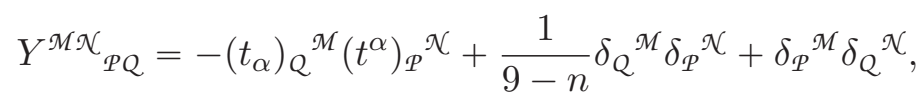

and thus the tensor $Z$ is

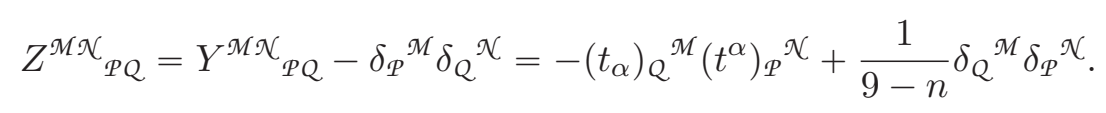

Comparing (3.13) with (2.16) we now find that

$$
f_{\mathscr{M}} \mathcal{X}_{P} Q+\tilde{f}_{\mathcal{M}} \mathcal{X}_{P} Q=-2 Z^{\mathcal{X} Q}{ }_{P \mathscr{M}},
$$

which can be inserted in the second term in the second line of (3.5), in order to express the generalized Lie derivative in terms of $\mathscr{B}_{n}$ and $\mathfrak{e}_{n+1}$. Although there is no need to simplify the first term on the right hand side of (3.5), it is interesting to note that it can be rewritten in a similar way, using

$$
f_{\mathcal{M}}^{\mathcal{X}_{P} Q}-\tilde{f}_{\mathcal{M}^{\mathcal{X}}}{ }_{P}^{Q}=-2 \delta_{\mathcal{M}} \mathcal{X}_{\delta_{P}}{ }^{Q} .
$$

The full expression for the generalized Lie derivative then becomes

$$
\begin{aligned}
& \mathscr{L}_{U} V^{Q}=-\frac{1}{2}\left(f_{\mathcal{M}^{\mathcal{X}}}{ }_{P}^{Q}-\tilde{f}_{\mathscr{M}^{\mathcal{X}}}{ }_{P}^{Q}\right) U^{\mathcal{M}} \partial_{\mathcal{X}} V^{P} \\
& -\frac{1}{2}\left(f_{\mathcal{M}} \mathcal{N}_{\mathcal{P}}{ }^{Q}+\tilde{f}_{\mathcal{M}} \mathcal{N}_{\mathcal{P}}{ }^{Q}\right) \partial_{\mathcal{X}} U^{\mathcal{M}} V^{\mathcal{P}} \text {. }
\end{aligned}
$$

Note that we get back the ordinary Lie derivative,

$$
L_{U} V^{m}=U^{n} \partial_{n} V^{m}-\partial_{n} U^{m} V^{n}
$$

from (3.16) by restricting $\mathscr{U}_{1}$ and $\tilde{\mathscr{U}}_{1}$ to $\mathscr{U}_{1}^{+}$and $\tilde{\mathscr{U}}_{1}^{+}$, respectively, and using (2.17).

By considering $\mathscr{B}_{n}$ and $\mathfrak{e}_{n+1}$ as subalgebras of $\mathscr{B}_{n+1}$ it is possible to obtain an expression where the components of the vector fields do not appear explicitly. If we set $\tilde{V}=\left[e_{-1}, V\right]=V^{\mathcal{M}} \tilde{E}_{\mathcal{M}}$ for any vector field $V$, then it follows from $(2.20)$ that

$$
\mathscr{L}_{U} V=\left[\left[U, \tilde{F}^{\mathcal{X}}\right], \partial_{\mathfrak{X}} \tilde{V}\right]-\left[\left[\partial_{\mathcal{N}} \tilde{U}, \tilde{F}^{\mathcal{X}}\right], V\right] .
$$

Applying the adjoint action of $e_{-1}$ to both sides of (3.18), and rewriting the right hand side using (2.20), we get the equivalent expression

$$
\mathscr{L}_{U} \tilde{V}=-\left[\left[\tilde{U}, F^{\mathcal{N}}\right], \partial_{\mathfrak{X}} V\right]-\left[\left[\partial_{\mathfrak{X}} U, F^{\mathcal{N}}\right], \tilde{V}\right],
$$

which turns out to be more useful in analyzing the reducibility of the generalized diffeomorphisms, as we will see in section 3.4.

The component-free expressions (3.18) and (3.19) make the reduction of exceptional geometry to ordinary geometry described above more clear. It simply amounts to restricting the vector fields $U$ and $V$, considered as elements in (the subspace $\mathscr{U}_{1}$ of) the Borcherds 
superalgebra $\mathscr{B}_{n}$, to the subalgebra $A(n-1,0)=\mathfrak{s l}(n \mid 1)$, obtained by removing node $n$ from the Dynkin diagram of $\mathscr{B}_{n}$. Similarly, we obtain the generalized Lie derivative in doubled geometry with T-duality group $\mathrm{O}(d, d)$, where $d=n-1$, from (3.18) by restricting the vector fields to the subalgebra $D(d, 1)=\mathfrak{o s p}(2 d \mid 2)$ corresponding to removing node $(n-1)$ from the Dynkin diagram of $\mathscr{B}_{n}$.

Another advantage of the expressions (3.18)-(3.19) is that the commutator of two generalized Lie derivatives can be computed using the Jacobi identity in $\mathscr{B}_{n+1}$. In practice it seems however easier to keep the expression (3.5) and use the identities (3.8)-(3.11), but as we will see in the next section, these identities can be derived from the Jacobi identity in $\mathscr{B}_{n+1}$, except for half of the identity (3.9), for which some additional information is needed.

\subsection{Closure}

The closure of the generalized diffeomorphisms into a Lie algebra relies on the identities (3.8)-(3.11) for the tensor $Y$, which we will now derive by expressing $Y$ in terms of $f$ and $\tilde{f}$. This can be done in various ways,

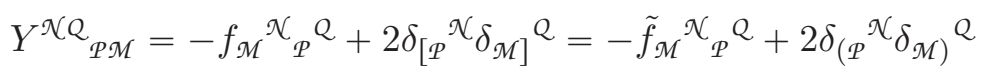

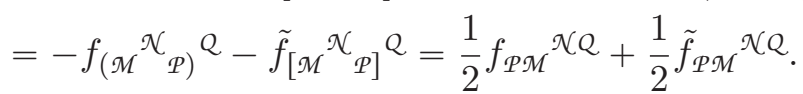

From the last expression it follows that (3.8) is equivalent to the section condition (3.3). We will show that this condition, together with the Jacobi identity in $\mathscr{B}_{n+1}$, implies (3.10), (3.11) and the part of (3.9) symmetric in the indices $\mathcal{R}$ and $\mathcal{S}$. To derive the part of (3.9) antisymmetric in $\mathcal{R}$ and $s$ we also need the fact that $\tilde{\mathscr{U}}_{ \pm 2}$ is at most one-dimensional for $n \leqslant 7$.

Let $\hat{\mathscr{U}}_{1}=\mathscr{U}_{1} \oplus \tilde{\mathscr{U}}_{1}$ be the level-one subspace of $\mathscr{B}_{n+1}$ in the level decomposition with respect to node 0 (the innermost of the two gray nodes) in the Dynkin diagram. Let $\tau$ be a vector space automorphism of $\mathscr{B}_{n+1}$ such that $\tau([x, y])=[\tau(y), \tau(x)]$, preserving the $\mathbb{Z}_{2}$-degree but reversing the $\mathbb{Z}$-degree, so that $\tau\left(\mathscr{U}_{ \pm 1}\right)=\mathscr{U}_{\mp 1}$ and $\tau\left(\tilde{\mathscr{U}}_{ \pm 1}\right)=\tilde{\mathscr{U}}_{\mp 1}$. As a consequence of the Jacobi identity in $\mathscr{B}_{n+1}$, the triple product

$$
\hat{\mathscr{U}}_{1} \times \hat{\mathscr{U}}_{1} \times \hat{\mathscr{U}}_{1} \rightarrow \hat{\mathscr{U}}_{1}, \quad(x, y, z) \mapsto(x y z) \equiv[[x, \tau(y)], z],
$$

then satisfies the identity

$$
(u v(x y z))-(-1)^{\sigma}(x y(u v z))=((u v x) y z)-(-1)^{\sigma}(x(v u y) z)
$$

where $\sigma=(|u|+|v|)(|x|+|y|)$, denoting the $\mathbb{Z}_{2}$-degree of any element $z$ by $|z|$. Indeed, the Jacobi identity turns the left hand side of (3.22) into

$$
[[[u, \tau(v)],[x, \tau(y)]], z],
$$

which after using the Jacobi identity once again becomes

$$
[[[[u, \tau(v)], x], \tau(y)], z]-(-1)^{|x||y|}[[[[u, \tau(v)], \tau(y)], x], z],
$$


where the last term is equal to

$$
-(-1)^{\sigma}[[x, \tau([[v, \tau(u)], y])], z] .
$$

We choose the linear map $\tau$ to be given by $\tau\left(E_{\mathscr{M}}\right)=F^{\mathscr{M}}$ and $\tau\left(\tilde{E}_{\mathscr{M}}\right)=\tilde{F}^{\mathscr{M}}$. According to $(2.14)$ we then have

$$
\left(E_{\mathcal{M}} E_{\mathcal{X}} E_{\mathcal{P}}\right)=f_{\mathcal{M}} \mathcal{X}_{\mathcal{P}}{ }^{Q} E_{Q}, \quad\left(\tilde{E}_{\mathcal{M}} \tilde{E}_{\mathcal{N}} \tilde{E}_{\mathcal{P}}\right)=\tilde{f}_{\mathcal{M}} \mathcal{X}_{\mathcal{P}}{ }^{Q} \tilde{E}_{\mathcal{Q}}
$$

for the basis elements $E_{\mathscr{M}}$ and $\tilde{E}_{\mathscr{M}}$ of $\mathscr{U}_{1}$ and $\tilde{\mathscr{U}}_{1}$, respectively, and thus these subspaces of $\hat{\mathscr{U}}_{1}$ close under the triple product (3.21). Since they are homogeneous with respect to the $\mathbb{Z}_{2}$-grading, the identity (3.22) on these subspaces becomes

$$
(x y(u v z))-(u v(x y z))=((x y u) v z)-(x(v u y) z)
$$

which means that they satisfy the definition of a generalized Jordan triple system [47]. For $E_{\mathcal{M}}$ the identity (3.27) can be written in component form as

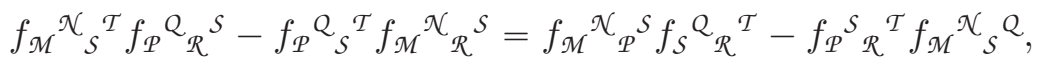

and for $\tilde{E}_{\mathscr{M}}$ the same identity holds with $f$ replaced with $\tilde{f}$.

It follows from (2.20) that also the subspace of $\hat{\mathscr{U}}_{1}$ spanned by all linear combinations $\hat{E}_{\mathscr{M}}=E_{\mathcal{M}}+\tilde{E}_{\mathcal{M}}$ closes under the triple product (3.21), which for these basis elements is

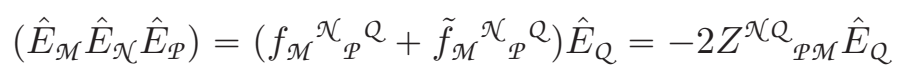

and the component form of the identity (3.22) for this subspace is again given by (3.28),

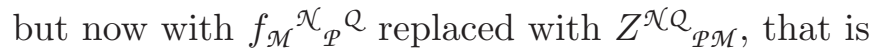

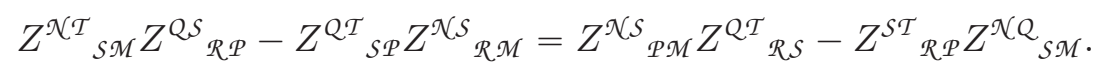

After symmetrizing (3.30) in the indices ${ }^{\mathcal{X}}$ and $\mathcal{Q}$ the left hand side is antisymmetric in the indices $\mathscr{M}_{\mathcal{M}}$ and ${ }_{\mathcal{P}}$, so the right hand side must be antisymmetric in $\mathcal{M}$ and ${ }_{\mathcal{P}}$ as well. Likewise, after antisymmetrizing (3.30) in $\mathcal{X}$ and $\mathcal{Q}$ the right hand side must be symmetric in $\mathscr{M}$ and ${ }_{p}$. Thus (3.30) is equivalent to the set of identities

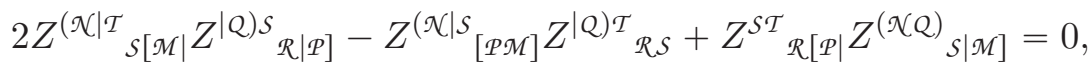

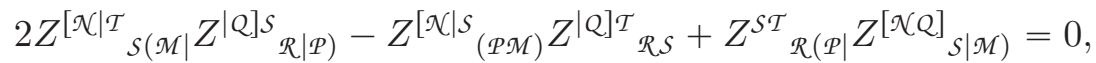

$$
\begin{aligned}
& Z^{(\mathcal{N} \mid S}{ }_{(\mathcal{P M})} Z^{\mid Q) \mathcal{T}}{ }_{R S}-Z^{\mathcal{S T}}{ }_{R(P \mid} Z^{(\mathcal{N} Q)}{ }_{S \mid \mathcal{M})}=0,
\end{aligned}
$$

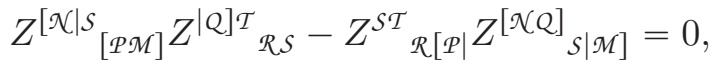

which, using $Z^{\mathfrak{M} \mathcal{N}_{P Q}}=Y^{\mathscr{M} \mathfrak{N}_{P Q}}-\delta_{P}{ }^{\mathscr{M}} \delta_{Q} \mathcal{X}$, can be written

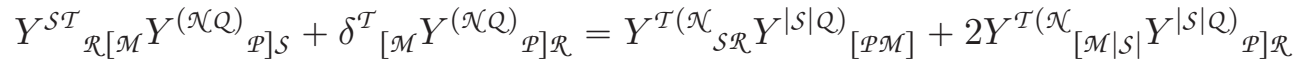

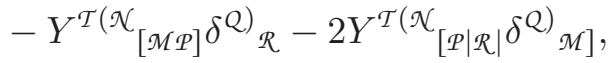




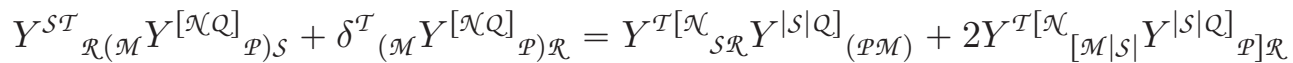

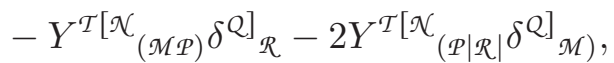

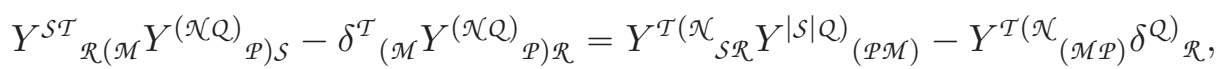

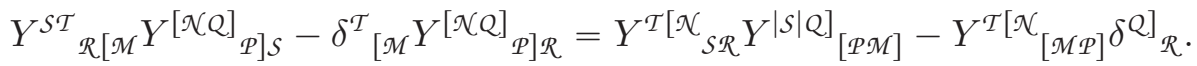

Contracting (3.32)-(3.34) with $\partial_{\mathcal{N}} \otimes \partial_{\mathcal{Q}}$ and using (3.8) the left hand sides vanishes and we get, respectively, (3.10), (3.11) and half of (3.9), namely the part symmetric in the indices $\mathcal{R}$ and $\mathcal{S}_{\mathcal{S}}\left(\right.$ here $_{\mathcal{P}}$ and $\mathscr{M}$ ). Doing the same with (3.35) gives the additional identity

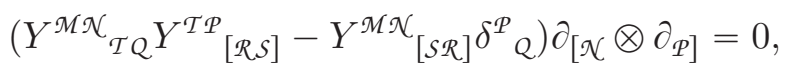

which is not needed for the closure of the generalized diffeomorphisms. (However, it is in fact needed for their covariance, $\mathscr{L}_{U} \mathscr{L}_{V}=\mathscr{L}_{\mathscr{L}_{U} V}+\mathscr{L}_{V} \mathscr{L}_{U}$.) The remaining antisymmetric part of (3.9) reads

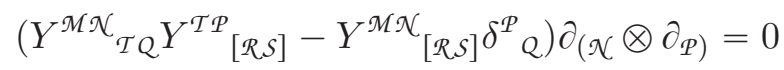

and is trivially satisfied for $n \leqslant 6$ since $Y$ then is symmetric in the lower (and upper) indices. It still holds for $n=7$ but fails for $n=8$. This can be understood from the $\mathbb{Z}$-grading of $\mathfrak{e}_{n+1}$ with respect to $\mathfrak{e}_{n}$. For $n \leqslant 6$ this is a 3 -grading,

$$
\left[\tilde{E}_{\mathcal{M}}, \tilde{E}_{\mathcal{N}}\right]=\left[\tilde{F}^{\mathcal{M}}, \tilde{F}^{\mathcal{N}}\right]=0,
$$

which is equivalent to the symmetry of $Y$ in its lower (and upper) indices, since

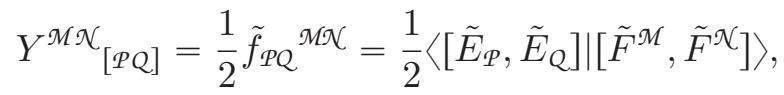

while for $n=7$ we have a 5 -grading of $\mathfrak{e}_{8}$ with respect to $\mathfrak{e}_{7}$, where the subspaces $\tilde{\mathscr{U}}_{ \pm 2}$ are one-dimensional. This means that $\left[\tilde{E}_{\mathcal{M}},\left[\tilde{F}^{\mathcal{N}},\left[\tilde{E}_{\mathscr{P}}, \tilde{E}_{Q}\right]\right]\right]$ must be proportional to $\delta_{\mathscr{M}} \mathcal{X}\left[\tilde{E}_{\mathcal{P}}, \tilde{E}_{Q}\right]$, and it is easy to check that this proportionality in fact is an equality. From this equality, and the 5 -grading,

$$
\left[\tilde{E}_{\mathcal{M}},\left[\tilde{E}_{\mathcal{N}}, \tilde{E}_{\mathcal{P}}\right]\right]=\left[\tilde{F}^{\mathcal{M}},\left[\tilde{F}^{\mathcal{N}}, \tilde{F}^{\mathcal{P}}\right]\right]=0,
$$

we get the identities

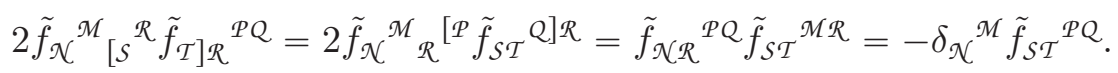

Expressed in $Y$ the last two equations become

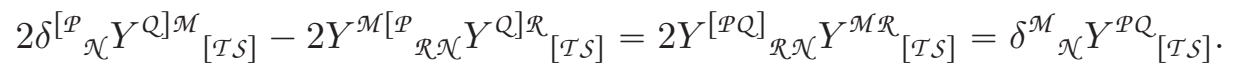

For the antisymmetric part of (3.9) we now get

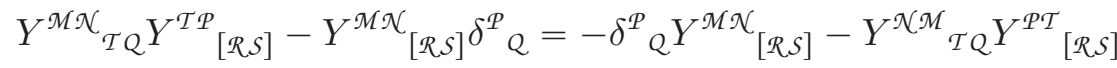

$$
\begin{aligned}
& -2 Y^{[\mathcal{M N}]_{\mathcal{T Q}} Y^{\mathcal{P T}}}[R S]
\end{aligned}
$$




$$
\begin{aligned}
& =2\left(\delta^{\left[\mathcal{M}_{Q} Y^{\mathcal{T}] \mathcal{K}}\right.}{ }_{[\mathcal{R} S]}-Y^{\mathcal{N}\left[\mathcal{M}_{\mathcal{T} Q} Y^{\mathcal{T}] \mathcal{T}}\right.}[\text { RS }]\right) \\
& -\delta^{\mathscr{M}}{ }_{Q} Y^{P \mathcal{N}}{ }_{[R S]}-Y^{\mathcal{X}{ }_{\mathcal{T} Q}} Y^{\mathcal{M T} \mathcal{T}}[\mathcal{R} S] \\
& -\delta^{P}{ }_{Q} Y^{\mathscr{M} \mathcal{K}}[R S] \\
& =-\delta^{\mathscr{M}}{ }_{Q} Y^{\mathscr{P \mathcal { K }}}{ }_{[\mathcal{R} S]}-Y^{\mathscr{N} \mathcal{P}}{ }_{\mathcal{T} Q} Y^{\mathscr{M T} \mathcal{T}}[\mathcal{R} S] \\
& -\delta^{\mathcal{P}}{ }_{Q} Y^{\mathcal{M} \mathcal{X}}{ }_{[\mathcal{R} S]}+\delta^{\mathcal{X}}{ }_{Q} Y^{\mathcal{M} P}{ }_{[\mathcal{R} S]},
\end{aligned}
$$

which after contraction with $\partial_{(\mathcal{N}} \otimes \partial_{\mathcal{P})}$ and using (3.8) gives (3.37).

\subsection{Reducibility}

We have seen that the possible transformations of a vector field $V$ under generalized diffeomorphisms are parametrized by vector fields $U$, which, as well as $V$, can be considered as elements in the subspace $\mathscr{U}_{1}$ of $\mathscr{B}_{n}$. However, the correspondence between all possible transformations of $V$ and all elements $U$ in $\mathscr{U}_{1}$ is not one-to-one. If $U$ is given by $U=\partial_{\mathscr{M}}\left[U^{\prime}, F^{\mathscr{M}}\right]$ for some $U^{\prime}$ in $\mathscr{U}_{2}$, then the Jacobi identity gives

$$
\left[\partial_{\mathscr{M}}\left[U, F^{\mathscr{M}}\right], \tilde{V}\right]=\left[\partial_{\mathscr{M}} \partial_{\mathcal{X}}\left[\left[U^{\prime}, F^{\mathcal{N}}\right], F^{\mathscr{M}}\right], \tilde{V}\right]=\frac{1}{2}\left[\partial_{\mathscr{M}} \partial_{\mathfrak{X}}\left[U^{\prime},\left[F^{\mathcal{N}}, F^{\mathscr{M}}\right]\right], \tilde{V}\right]
$$

for the second term in (3.19), or (4.2) below. This vanishes by the section condition since $\left[F^{\mathcal{N}}, F^{\mathcal{M}}\right]$ belongs to $\mathscr{U}_{-2}$, and thus project $\partial_{\mathcal{M}} \partial_{\mathfrak{K}}$ on $R_{2}$. Similarly we get

$$
\left[\left[\tilde{U}, F^{\mathscr{M}}\right], \partial_{\mathscr{M}} V\right]=\left[\partial_{\mathcal{N}}\left[\left[\tilde{U}^{\prime}, F^{\mathcal{N}}\right], F^{\mathscr{M}}\right], \partial_{\mathscr{M}} V\right]=\frac{1}{2}\left[\partial_{\mathscr{N}}\left[\tilde{U}^{\prime},\left[F^{\mathcal{N}}, F^{\mathcal{M}}\right]\right], \partial_{\mathscr{M}} V\right]
$$

for the first term (the transport term), which also vanishes. Thus any element $U$ in $\mathscr{U}_{1}$ given by $U=\partial_{\mathscr{M}}\left[U^{\prime}, F^{\mathscr{M}}\right]$ for some $U^{\prime}$ in $\mathscr{U}_{2}$ generates a zero transformation. However, the correspondence between elements in $\mathscr{U}_{1}$ that generate zero transformations and general elements in $\mathscr{U}_{2}$ is not one-to-one either, since $\partial_{\mathscr{M}}\left[U^{\prime}, F^{\mathscr{M}}\right]=0$ if $U^{\prime}$ is given by $U^{\prime}=$ $\partial_{\mathscr{M}}\left[U^{\prime \prime}, F^{\mathscr{M}}\right]$ for some $U^{\prime \prime}$ in $\mathscr{U}_{3}$. Continuing in this way the naive counting for the effective number of parameters is given by the alternating sum

$$
\operatorname{dim} \mathscr{U}_{1}-\operatorname{dim} \mathscr{U}_{2}+\operatorname{dim} \mathscr{U}_{3}-\operatorname{dim} \mathscr{U}_{4}+\cdots
$$

which is highly divergent since the dimensions of $\mathscr{U}_{p}$ increase with the level $p$. In [38] it was shown, using a corresponding partition function, that the alternating sum (3.46) can be regularized to give the correct number coming from the decomposition of generalized diffeomorphisms into ordinary diffeomorphisms, 2- and 5-form gauge transformations, and dual diffeomorphisms. The corresponding partition function is related to the denominator formula for $\mathscr{B}_{n}$, as shown in [39].

Introducing an operator $\partial=\left(\operatorname{ad} F^{\mathcal{M}}\right) \partial_{\mathscr{M}}$ the alternating sum (3.46) can be viewed as expressing the homology of the chain complex

$$
\mathscr{U}_{1} \stackrel{\partial}{\longleftarrow} \mathscr{U}_{2} \stackrel{\partial}{\longleftarrow} \mathscr{U}_{3} \stackrel{\partial}{\longleftarrow} \mathscr{U}_{4} \stackrel{\partial}{\longleftarrow} \cdots
$$

where the nilpotency $\partial^{2}=0$ follows from the section condition, again by the Jacobi identity. In [12] it was shown that the derivative in the operator $\partial$ from $\mathscr{U}_{p+1}$ to $\mathscr{U}_{p}$ is covariant only for $1 \leqslant p \leqslant 7-n$. 
The infinite reducibility of the generalized diffeomorphisms in exceptional geometry is a qualitative difference compared to ordinary and doubled geometry. As explained in section 3.2 , these cases can be obtained from the exceptional one by restricting $\mathscr{B}_{n}$ to the subalgebras $A(n-1,0)=\mathfrak{s l}(n \mid 1)$ and $D(n-1,1)=\mathfrak{o s p}(2 n-2 \mid 2)$, obtained by removing node $n$ and node $(n-1)$ from the Dynkin diagram of $\mathscr{B}_{n}$, respectively. With respect to the gray node, $A(n-1,0)$ is 3 -graded and $D(n-1,1)$ is 5 -graded, which implies no reducibility in ordinary geometry and a first-order finite reducibility in double geometry.

\section{Discussion}

In this paper we have studied generalized diffeomorphisms in exceptional geometry with U-duality group $E_{n(n)}$ for $n \leqslant 7$. By considering any vector field $V$ as an element in the level-one subspace $\mathscr{U}_{1}$ of the Borcherds superalgebra $\mathscr{B}_{n}$, which in turn is considered as a subalgebra of $\mathscr{B}_{n+1}$, we have found that the generalized Lie derivative of $V$ parametrized by another vector field $U$ can be written

$$
\mathscr{L}_{U} V=\left[\left[U, \tilde{F}^{\mathcal{X}}\right], \partial_{\mathfrak{X}} \tilde{V}\right]-\left[\left[\partial_{\mathcal{X}} \tilde{U}, \tilde{F}^{\mathcal{X}}\right], V\right],
$$

or equivalently

$$
\mathscr{L}_{U} \tilde{V}=-\left[\left[\tilde{U}, F^{\mathcal{N}}\right], \partial_{\mathfrak{X}} V\right]-\left[\left[\partial_{\mathfrak{X}} U, F^{\mathcal{N}}\right], \tilde{V}\right],
$$

where the tilde on $U$ and $V$ denotes the adjoint action of $e_{-1}$, and $\tilde{F}^{\mathscr{M}}=-\left[f_{-1}, F^{\mathscr{M}}\right]$. It would of course be interesting to include also other aspects of exceptional geometry in this framework, for example concepts of connection, torsion of curvature which could be needed for an extension to the case $n=8$ (and beyond). The transformations (4.1) and (4.2) can be defined in precisely the same way for $n=8$ as for $n \leqslant 7$, but then they fail to close, as was noted in [38]. We have shown in this paper that the failure can be understood from the $\mathbb{Z}$-grading of $\mathfrak{e}_{9}$ which respect to $\mathfrak{e}_{8}$. With the notation in [21] we only get $\dot{\mathscr{L}}_{U} V$ from the right hand side of (4.1) and we have to supplement this expression with an additional term, involving an additional 'section-projected' parameter field, in order to obtain closed and covariant transformations [18, 20,21].

An advantage of the expressions (4.1) and (4.2) is their universality. They are at the same time valid not only for any $D \geqslant 4$, but also for ordinary geometry and doubled geometry as well as for exceptional geometry. The cases of ordinary and doubled geometry can be obtained from the exceptional case by simply restricting $\mathscr{B}_{n+1}$ to subalgebras, corresponding to removing nodes from the Dynkin diagram, without changing the expression for the generalized Lie derivative. However, it seems unsatisfactory from the universality point of view that different algebras $\mathscr{B}_{n+1}$ are needed for different $n$. This problem can probably be solved by taking into account also the $D$ external dimensions and adding $D-1$ white nodes to the $\mathscr{B}_{n+1}$ diagram so that the additional white nodes form a Dynkin diagram of $\mathfrak{a}_{D-1}=\mathfrak{s l}(D)$, connected with a line from one of its end nodes to the outermost gray node in the $\mathscr{B}_{n+1}$ diagram. The different Dynkin diagrams

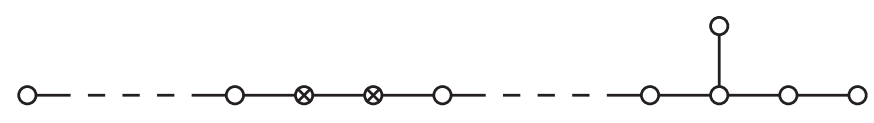

obtained in this way for different $D$ are in fact equivalent in the sense that they describe the same Borcherds superalgebra $\mathscr{B}_{11}$. By a series of odd reflections a 'distinguished' Dynkin 
diagram can be reached, with only one gray node connected to the Dynkin diagram of $\mathfrak{e}_{11}$. If our results can be extended accordingly they could be related to the $\mathfrak{e}_{11}$ approach in $[7,48-50]$.

The partial derivatives in (4.2) appear contracted with the basis elements $F^{\mathscr{M}}$ of $\mathscr{U}_{-1}$, and as a consequence, the section condition can be relaxed if the general basis elements $F^{\mathscr{M}}$ are replaced by elements $\Phi^{\mathcal{M}}$ that are not linearly independent, but span a subspace of $\mathscr{U}_{-1}$ (equivalent to $\mathscr{U}_{-1}{ }^{0}$ for the solutions corresponding to eleven-dimensional supergravity) which is 'isotropic' in the sense that $\left[\Phi^{\mathcal{M}}, \Phi^{\mathcal{N}}\right]=0$ for any pair of elements $\Phi^{\mathcal{M}}$ and $\Phi^{\mathcal{K}}$. This alternative version of the section condition might be easier to handle than the original one, and might be especially useful in further work connecting exceptional geometry to gauged supergravity (possibly related to the previous work $[14,15,19]$ ), since the truncation of $\mathscr{B}_{n+1}$ given by the projection of $F^{\mathscr{M}}$ on the elements $\Phi^{\mathscr{M}}$ satisfying $\left[\Phi^{\mathcal{M}}, \Phi^{\mathcal{N}}\right]=0$ is similar to the truncation of the tensor hierarchy algebra [30], where the subspace at level -1 is spanned by the embedding tensor $\Theta$, satisfying the quadratic constraint $[\Theta, \Theta]=0$.

\section{Acknowledgments}

I would like to thank Martin Cederwall, Sebastian Guttenberg, Axel Kleinschmidt and Ergin Sezgin for valuable discussions, and the referee for making me aware of a few typos. The work is supported by NSF grant PHY-1214344.

Open Access. This article is distributed under the terms of the Creative Commons Attribution License (CC-BY 4.0), which permits any use, distribution and reproduction in any medium, provided the original author(s) and source are credited.

\section{References}

[1] C.M. Hull, Generalised geometry for M-theory, JHEP 07 (2007) 079 [hep-th/0701203] [INSPIRE].

[2] P.P. Pacheco and D. Waldram, M-theory, exceptional generalised geometry and superpotentials, JHEP 09 (2008) 123 [arXiv:0804.1362] [INSPIRE].

[3] C. Hillmann, Generalized $E_{7(7)}$ coset dynamics and $D=11$ supergravity, JHEP 03 (2009) 135 [arXiv:0901.1581] [INSPIRE].

[4] D.S. Berman and M.J. Perry, Generalized geometry and M-theory, JHEP 06 (2011) 074 [arXiv: 1008.1763] [INSPIRE].

[5] D.S. Berman, H. Godazgar and M.J. Perry, $\mathrm{SO}(5,5)$ duality in M-theory and generalized geometry, Phys. Lett. B 700 (2011) 65 [arXiv:1103.5733] [INSPIRE].

[6] D.S. Berman, H. Godazgar, M. Godazgar and M.J. Perry, The local symmetries of M-theory and their formulation in generalised geometry, JHEP 01 (2012) 012 [arXiv:1110.3930] [INSPIRE].

[7] D.S. Berman, H. Godazgar, M.J. Perry and P. West, Duality invariant actions and generalised geometry, JHEP 02 (2012) 108 [arXiv:1111.0459] [INSPIRE].

[8] A. Coimbra, C. Strickland-Constable and D. Waldram, $E_{d(d)} \times \mathbb{R}^{+}$generalised geometry, connections and M-theory, JHEP 02 (2014) 054 [arXiv: 1112.3989] [INSPIRE]. 
[9] A. Coimbra, C. Strickland-Constable and D. Waldram, Supergravity as generalised geometry II: $E_{d(d)} \times \mathbb{R}^{+}$and M-theory, JHEP 03 (2014) 019 [arXiv: 1212.1586] [INSPIRE].

[10] J.-H. Park and Y. Suh, U-gravity: SL(N), JHEP 06 (2014) 102 [arXiv:1402.5027] [INSPIRE].

[11] J.-H. Park and Y. Suh, U-geometry: SL(N), JHEP 04 (2013) 147 [Erratum ibid. 11 (2013) 210] [arXiv: 1302.1652] [INSPIRE].

[12] M. Cederwall, J. Edlund and A. Karlsson, Exceptional geometry and tensor fields, JHEP 07 (2013) 028 [arXiv:1302.6736] [INSPIRE].

[13] M. Cederwall, Non-gravitational exceptional supermultiplets, JHEP 07 (2013) 025 [arXiv: 1302.6737] [INSPIRE].

[14] G. Aldazabal, M. Graña, D. Marqués and J.A. Rosabal, Extended geometry and gauged maximal supergravity, JHEP 06 (2013) 046 [arXiv: 1302.5419] [INSPIRE].

[15] G. Aldazabal, M. Graña, D. Marqués and J.A. Rosabal, The gauge structure of exceptional field theories and the tensor hierarchy, JHEP 04 (2014) 049 [arXiv: 1312.4549] [INSPIRE].

[16] O. Hohm and H. Samtleben, Exceptional field theory I: $E_{6(6)}$ covariant form of M-theory and type IIB, Phys. Rev. D 89 (2014) 066016 [arXiv:1312.0614] [INSPIRE].

[17] O. Hohm and H. Samtleben, Exceptional field theory II: $E_{7(7)}$, Phys. Rev. D 89 (2014) 066017 [arXiv: 1312.4542] [INSPIRE].

[18] O. Hohm and H. Samtleben, Exceptional field theory III: $E_{8(8)}$, Phys. Rev. D 90 (2014) 066002 [arXiv:1406.3348] [InSPIRE].

[19] O. Hohm and H. Samtleben, Consistent Kaluza-Klein truncations via exceptional field theory, JHEP 01 (2015) 131 [arXiv:1410.8145] [INSPIRE].

[20] J.A. Rosabal, On the exceptional generalised Lie derivative for $d \geqslant 7$, JHEP 09 (2015) 153 [arXiv: 1410.8148] [INSPIRE].

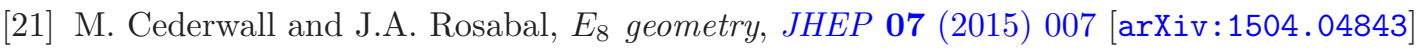
[INSPIRE].

[22] E. Cremmer, B. Julia, H. Lü and C.N. Pope, Dualization of dualities II: twisted self-duality of doubled fields and superdualities, Nucl. Phys. B 535 (1998) 242 [hep-th/9806106] [INSPIRE].

[23] P. Henry-Labordere, B. Julia and L. Paulot, Borcherds symmetries in M-theory, JHEP 04 (2002) 049 [hep-th/0203070] [INSPIRE].

[24] P. Henry-Labordere, B. Julia and L. Paulot, Real Borcherds superalgebras and M-theory, JHEP 04 (2003) 060 [hep-th/0212346] [INSPIRE].

[25] M. Henneaux, B.L. Julia and J. Levie, $E_{11}$, Borcherds algebras and maximal supergravity, JHEP 04 (2012) 078 [arXiv: 1007.5241] [INSPIRE].

[26] J. Greitz and P.S. Howe, Maximal supergravity in $D=10$ : forms, Borcherds algebras and superspace cohomology, JHEP 08 (2011) 146 [arXiv:1103.5053] [INSPIRE].

[27] J. Greitz and P.S. Howe, Half-maximal supergravity in three dimensions: supergeometry, differential forms and algebraic structure, JHEP 06 (2012) 177 [arXiv:1203.5585] [INSPIRE].

[28] J. Greitz, P. Howe and J. Palmkvist, The tensor hierarchy simplified, Class. Quant. Grav. 31 (2014) 087001 [arXiv:1308.4972] [InSPIRE].

[29] P. Howe and J. Palmkvist, Forms and algebras in (half-)maximal supergravity theories, JHEP 05 (2015) 032 [arXiv:1503.00015] [INSPIRE]. 
[30] J. Palmkvist, The tensor hierarchy algebra, J. Math. Phys. 55 (2014) 011701 [arXiv: 1305.0018] [INSPIRE].

[31] F. Riccioni and P.C. West, The $E_{11}$ origin of all maximal supergravities, JHEP 07 (2007) 063 [arXiv:0705.0752] [INSPIRE].

[32] E.A. Bergshoeff, I. De Baetselier and T.A. Nutma, $E_{11}$ and the embedding tensor, JHEP 09 (2007) 047 [arXiv: 0705.1304] [INSPIRE].

[33] E.A. Bergshoeff, J. Gomis, T.A. Nutma and D. Roest, Kac-Moody spectrum of (half-)maximal supergravities, JHEP 02 (2008) 069 [arXiv:0711.2035] [INSPIRE].

[34] F. Riccioni and P.C. West, $E_{11}$-extended spacetime and gauged supergravities, JHEP 02 (2008) 039 [arXiv:0712.1795] [INSPIRE].

[35] P.C. West, $E_{11}$ and M-theory, Class. Quant. Grav. 18 (2001) 4443 [hep-th/0104081] [INSPIRE].

[36] J. Palmkvist, Tensor hierarchies, Borcherds algebras and E $E_{11}$, JHEP 02 (2012) 066 [arXiv: 1110.4892] [INSPIRE].

[37] J. Palmkvist, Borcherds and Kac-Moody extensions of simple finite-dimensional Lie algebras, JHEP 06 (2012) 003 [arXiv:1203.5107] [INSPIRE].

[38] D.S. Berman, M. Cederwall, A. Kleinschmidt and D.C. Thompson, The gauge structure of generalised diffeomorphisms, JHEP 01 (2013) 064 [arXiv: 1208.5884] [INSPIRE].

[39] M. Cederwall and J. Palmkvist, Superalgebras, constraints and partition functions, JHEP 08 (2015) 036 [arXiv: 1503.06215] [INSPIRE].

[40] B. de Wit and H. Samtleben, Gauged maximal supergravities and hierarchies of non-Abelian vector-tensor systems, Fortsch. Phys. 53 (2005) 442 [hep-th/0501243] [INSPIRE].

[41] B. de Wit, H. Nicolai and H. Samtleben, Gauged supergravities, tensor hierarchies and M-theory, JHEP 02 (2008) 044 [arXiv:0801.1294] [INSPIRE].

[42] V.G. Kac, Lie superalgebras, Adv. Math. 26 (1977) 8 [INSPIRE].

[43] V.G. Kac, Infinite dimensional Lie algebras, $3^{\text {rd }}$ edition, Cambridge University Press, Cambridge U.K. (1990).

[44] M. Wakimoto, Infinite-dimensional Lie algebras, American Mathematical Society, U.S.A. (2001).

[45] U. Ray, Automorphic forms and Lie superalgebras, Springer, Dordrecht The Netherlands (2006).

[46] A. Kleinschmidt and J. Palmkvist, Oxidizing Borcherds symmetries, JHEP 03 (2013) 044 [arXiv:1301.1346] [INSPIRE].

[47] I.L. Kantor, Some generalizations of Jordan algebras, Trudy Sem. Vect. Tens. Anal. 16 (1972) 407.

[48] P. West, Generalised geometry, eleven dimensions and $E_{11}, J H E P 02$ (2012) 018 [arXiv: 1111.1642] [INSPIRE].

[49] P. West, $E_{11}$, generalised space-time and equations of motion in four dimensions, JHEP 12 (2012) 068 [arXiv:1206.7045] [InSPIRE].

[50] P. West, Generalised space-time and gauge transformations, JHEP 08 (2014) 050 [arXiv: 1403.6395] [INSPIRE]. 